\title{
Prism-coupled surface wave accelerator based on silicon carbide
}

\author{
Burton Neuner III, ${ }^{1}$ Dmitriy Korobkin, ${ }^{1}$ Gabriel Ferro, ${ }^{2}$ and Gennady Shvets ${ }^{1, *}$ \\ ${ }^{1}$ Department of Physics, University of Texas at Austin, 1 University Station C1500, Austin, Texas 78712, USA \\ ${ }^{2}$ LMI, UMR-CNRS 5615, Université Claude Bernard Lyon 1, 69622 Villeurbanne, France
}

(Received 12 January 2012; published 27 March 2012)

\begin{abstract}
A compact, solid-state accelerating structure based on surface waves is proposed and experimentally characterized. The structure, consisting of two SiC layers epitaxially grown on Si slabs and separated from each other by a subwavelength acceleration channel, is shown to support both longitudinal (accelerating) and transverse (deflecting) surface modes. Both modes are experimentally excited and characterized by performing angle-resolved spectroscopy with a wavelength-tunable carbon dioxide laser. Phase velocities of superluminous and subluminous modes are experimentally demonstrated, paving the way for tabletop charged particle acceleration. The same structure can be used for optical characterization of ultrashort electron bunches that generate longitudinal and transverse Cherenkov radiation.
\end{abstract}

DOI: 10.1103/PhysRevSTAB.15.031302

PACS numbers: 29.20.Ej, 41.20.Jb, 42.82.Et, 71.36.+c

\section{INTRODUCTION}

Accelerating gradients of conventional accelerators consisting of microwave-driven metal cavities are severely limited by electric breakdown and available peak power [1]. Theoretical, computational, and technological (both in microfabrication and laser technology) advances have resulted in the resurgence of interest in advanced laser-driven structure-based accelerators that have been known [2] for some time. For example, modern lasers $[3,4]$ can deliver sufficient power to excite large gradient $(>100 \mathrm{MV} / \mathrm{m})$ solid-state accelerator structures [2]. Breakdown issues can be mitigated in dielectric waveguide accelerators [5] by either placing a smooth metallic confinement wall far away from the beam [6,7], or by avoiding metallic confinement altogether in favor of photonic band gap (PBG) confinement [8-12].

Most two- [11] and three-dimensional [8,9,12] dielectric PBG accelerators have been designed to operate at visible/ near-IR laser wavelengths, where considerable fabrication challenges must be overcome. Using longer-wavelength [13] (e.g., $\lambda=10.6 \mu \mathrm{m}$ ) laser drivers for dielectric accelerators would considerably mitigate fabrication challenges, as well as take advantage of the short-pulse (picosecond-scale) high-power (terawatt-scale) $\mathrm{CO}_{2}$ lasers that have recently become available at several experimental facilities [3,4]. Despite considerable theoretical effort in the area of advanced laser-driven structure-based accelerators, there has been relatively modest experimental progress, which is mostly confined to metal-based PBG [14] structures.

\footnotetext{
*gena@physics.utexas.edu
}

Published by the American Physical Society under the terms of the Creative Commons Attribution 3.0 License. Further distribution of this work must maintain attribution to the author(s) and the published article's title, journal citation, and DOI.
Recently, an alternative accelerator structure driven by a $\mathrm{CO}_{2}$ laser has been proposed $[15,16]$. The surface wave accelerator based on SiC (SWABSiC), shown in Fig. 1, utilizes a polar semiconductor (silicon carbide) that supports infrared surface waves at the $\mathrm{SiC} /$ vacuum interface because its dielectric permittivity $\epsilon_{\mathrm{SiC}}$ is negative within the tuning range of the $\mathrm{CO}_{2}$ laser. Below we report the first fabrication and optical characterization of the SWABSiC. Note that the SWABSiC structure, schematically shown in Fig. 2, can be employed as (i) an accelerator (in which case an external laser beam is needed to supply energy to the accelerated beam), or as (ii) a radiation source that uses a prebunched electron beam to generate coherent mid-IR Cherenkov radiation. Application (ii) can be potentially useful as a diagnostic tool for high-energy electron bunches. Specifically, the angular and spectral distributions of the coherent IR radiation outcoupled through the prism can be used to characterize the bunch length and transverse size. Such diagnostics are conceptually similar to the one based on coherent off-axis undulator radiation from short electron bunches [17].

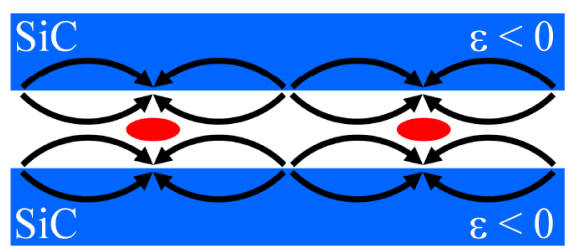

FIG. 1. Schematic of the surface wave accelerator based on $\mathrm{SiC}$ (SWABSiC). Acceleration occurs inside the vacuum gap between two parallel silicon carbide plates. Field lines extend from the surface of the SiC/vacuum interface into the channel and accelerate particle bunches (two bunches spaced by one acceleration wavelength are shown). Surface waves are localized near $\mathrm{SiC} /$ vacuum interfaces. 


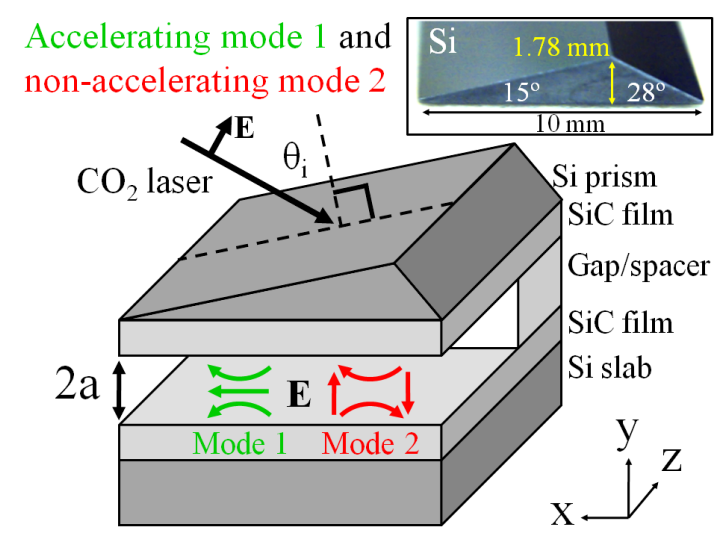

FIG. 2. Schematic of the SiC-based accelerator (not to scale). Thin layers of $\mathrm{SiC}$ are grown on $\mathrm{Si}$ slabs that can be fabricated into prisms. The prism couples $p$-polarized (TM) mid-IR radiation to the structure. A microfabricated spacer (one side shown) defines the vacuum gap for the beam accelerated by electric fields (E, depicted by arrows) parallel to the $\mathrm{SiC} /$ vacuum interfaces. Inset: a photograph of the $\mathrm{Si}$ coupling prism. The fabricated sample has a $2 a=6 \mu \mathrm{m}$ gap, $1.7-\mu \mathrm{m}$-thick SiC films, 5-mm-thick Si slabs, and prism dimensions as defined in the inset.

\section{MATERIAL PROPERTIES OF SILICON CARBIDE}

The schematic of the SWABSiC structure is shown in Fig. 1. The structure consists of two slabs of $\mathrm{SiC}$ separated by a narrow vacuum gap of width $2 a$. Electrons propagating inside the gap parallel to the $\mathrm{SiC} /$ vacuum interface are accelerated by the longitudinal component $E_{x}$ of the electric field of the surface wave. In general, surface waves exist at the interface between two media with opposite signs of the dielectric permittivity $\epsilon$. Because $\mathrm{SiC}$ is a polar semiconductor [18], it has a dielectric permittivity $\epsilon_{\mathrm{SiC}}<0$ in the mid-IR reststrahlen band, which overlaps with the tunability range of $\mathrm{CO}_{2}$ lasers $(9.6 \mu \mathrm{m}<\lambda<$ $11.3 \mu \mathrm{m}$ ) that are capable of providing high-power pulses on picosecond time scales $[3,4]$. The specific doubleinterface surface wave mode employed in the SWABSiC is similar to that occurring at the plasma/vacuum interface in a hollow channel plasma wakefield accelerator [19], and, unlike a single-interface surface wave, can have a luminous phase velocity $v_{\mathrm{ph}} \equiv \omega / k_{x}=c$ that is required for accelerating ultrarelativistic electrons.

Additional attractive features of $\mathrm{SiC}$, which are presently utilized in $\mathrm{rf}$ devices operating in hostile and high-temperature $\left(>1000^{\circ} \mathrm{C}\right)$ environments [20], are its robustness to heat loading and its high electrical breakdown (DC threshold $\sim 300 \mathrm{MV} / \mathrm{m}$ ). High-quality $\mathrm{SiC}$ films can be grown on silicon [21] and other substrates [22] that are subsequently machined into a prismlike shape for coupling infrared radiation into and out of the structure, as shown in Fig. 2. The number of free carriers in $\mathrm{SiC}$ is another important issue for the material's suitability for accelerator applications. It has been recently demonstrated [23] that doping levels of SiC (and, therefore, the density of free electrons) can be essentially eliminated by controlling the growth procedure. In the absence of free carriers, the frequency-dependent dielectric permittivity is given by

$$
\epsilon_{\mathrm{SiC}}(\omega)=\epsilon_{\mathrm{SiC}}^{\prime}+i \epsilon_{\mathrm{SiC}}^{\prime \prime}=\epsilon_{\infty} \frac{\omega^{2}-\omega_{\mathrm{LO}}^{2}+i \gamma \omega}{\omega^{2}-\omega_{\mathrm{TO}}^{2}+i \gamma \omega},
$$

where [24] $\epsilon_{\infty}=6.7$, the longitudinal optical phonon frequency $\omega_{\mathrm{LO}}=970 \mathrm{~cm}^{-1} \quad\left(\lambda_{\mathrm{LO}}=10.31 \mu \mathrm{m}\right)$, the transverse optical phonon frequency $\omega_{\mathrm{TO}}=794 \mathrm{~cm}^{-1}$ $\left(\lambda_{\mathrm{TO}}=12.59 \mu \mathrm{m}\right)$, and the damping rate due to vibrational anharmonicity $\gamma=4.75 \mathrm{~cm}^{-1}$. The reststrahlen band defined by $\epsilon_{\mathrm{SiC}}^{\prime}<0$ corresponds to $\lambda_{\mathrm{LO}}<\lambda<\lambda_{\mathrm{TO}}$ (where $\lambda=2 \pi / k_{0}$ and $k_{0}=\omega / c$ ).

\section{ELECTROMAGNETIC PROPERTIES OF THE SWABSiC}

The dispersion properties of the electromagnetic modes of an idealized SWABSiC comprised of a vacuum accelerating channel of width $2 a$ between two infinitely thick $\mathrm{SiC}$ walls are reviewed below. Two transverse magnetic (TM) modes, classified according to the symmetry of their magnetic field $H_{z}$ with respect to the midplane at $y=0$, can interact with the beam: an antisymmetric accelerating (longitudinal) mode (L-mode) with $H_{z}(+y)=-H_{z}(-y)$, and a symmetric deflecting (transverse) mode (T-mode) with $H_{z}(+y)=H_{z}(-y)$. From the standpoint of accelerator applications, the T-mode is the equivalent of transverse dipole modes in conventional accelerators which, when excited by a bunched beam, can lead to beam-breakup (BBU) instabilities and beam loss. The accelerating and deflecting components of the electric field of either mode are expressed in term of $H_{z}$ according to

$$
E_{x}=(c / i \omega \epsilon)\left(\partial H_{z} / \partial y\right), \quad E_{y}=-\left(c k_{x} / \omega \epsilon\right) H_{z},
$$

respectively, and all fields are assumed to be proportional to $\exp \left(i k_{x} x-i \omega t\right)$. The two guided TM modes can be interpreted as a result of hybridization between two surface phonon-polaritons supported by the individual $\mathrm{SiC} / \mathrm{vac}-$ uum interfaces [25].

To derive the modes' dispersion equations relating the complex propagation constant $k_{x}=k_{x}^{\prime}+i k_{x}^{\prime \prime}$ (longitudinal wave number and attenuation rate, respectively) to the real frequency $\omega \equiv k_{0} c$, the continuity of $H_{z}$ and $E_{x}$ is enforced at the interfaces $(y= \pm a)$. The spatial profiles for $0<y<a$ of the L-mode and T-mode are given by $H_{z}^{(L)}(y)=\sin (u y) / \sin (u a)$ and $H_{z}^{(T)}(y)=\cos (u y) / \cos (u a)$, respectively, and $H_{z}^{(L, T)}(y)=e^{-q(y-a)}$ for $y>a$. The transverse wave numbers $u$ and $q$ satisfy the dispersion relations in vacuum and $\mathrm{SiC}$, respectively: $u^{2}=k_{0}^{2}-k_{x}^{2}$ and $q^{2}=$ $k_{x}^{2}-k_{0}^{2} \epsilon_{\mathrm{SiC}}$. Applying the boundary conditions yields the following transcendental equations for complex $u^{(L, T)}$ (superscript denotes L- and T-modes): 


$$
\begin{aligned}
\frac{\tan \left(u^{(L)} a\right)}{u^{(L)} a} & =\frac{-\epsilon_{\mathrm{SiC}}}{\sqrt{R^{2}-\left(u^{(L)} a\right)^{2}}}, \\
\frac{1}{\tan \left(u^{(T)} a\right) u^{(T)} a} & =\frac{\epsilon_{\mathrm{SiC}}}{\sqrt{R^{2}-\left(u^{(T)} a\right)^{2}}},
\end{aligned}
$$

where $R^{2} \equiv\left(1-\epsilon_{\mathrm{SiC}}\right) k_{0}^{2} a^{2}$. The value of $u$ determines whether the modes are luminous, superluminous, or subluminous. If $\mathrm{SiC}$ losses are neglected, then $u=0$ corresponds to luminous modes, real $u$ corresponds to superluminous modes, and imaginary $u$ corresponds to subluminous modes. It can be shown from Eqs. (3) that, depending on the frequency, the L-mode can be either luminous, superluminous, or subluminous, whereas the T-mode cannot be luminous and, for narrow vacuum gaps with $2 a<\pi / k_{0}$, can only be subluminous.

The L-mode can be used for direct laser acceleration whenever $\operatorname{Re}\left(k_{x}\right)>k_{0}$, or $k_{0} a>k_{0} a_{\text {lum }} \equiv-\epsilon_{\mathrm{SiC}}^{\prime} / \sqrt{1-\epsilon_{\mathrm{SiC}}^{\prime}}$. The equality corresponds to luminous waves, and the inequality to the subluminous waves. Note that a subluminous wave with $v_{\mathrm{ph}}<c$ can also accelerate relativistic electrons whose velocity $v_{b} \approx c$ lies in the $x-z$ plane and makes the frequency-dependent Cherenkov angle $\theta_{b}=$ $\cos ^{-1}\left(v_{\mathrm{ph}} / v_{b}\right)$ with the direction of the phase velocity of the wave. Conversely, if the SWABSiC is used as a radiation source, a broad spectrum of L-modes (longitudinal wakes) satisfying the Cherenkov condition will be emitted at their corresponding angles $\theta_{b}(\omega)$ with respect to the direction of the electron bunch's velocity. An important disadvantage of utilizing subluminous waves for particle acceleration is that the beam, while being accelerated, will be also deflected in the $x-z$ plane from its path and, therefore, requires additional in-plane magnetic focusing. No beam deflection will be produced by the luminous L-mode, which has an additional advantage of uniform accelerating field: $E_{x}(y)=$ const across the vacuum gap. Because the ratio of the peak electric fields at the surface to that at the electron's location is given by $E_{y}(y=a) / E_{x}(y=0)=$ $\pi(2 a / \lambda)$, subwavelength gaps $(2 a<\lambda)$ are advantageous for particle acceleration. Narrow gaps impose an upper wavelength limit on luminous and subluminous modes according to $\left(-\epsilon_{\mathrm{SiC}}^{\prime}\right)<k_{0}^{2} a^{2}\left(1+\sqrt{1+4 / k_{0}^{2} a^{2}}\right) / 2$ because, according to Eq. (1), $-\epsilon_{\mathrm{SiC}}^{\prime}(\omega)$ is a monotonically growing function of the wavelength $\lambda=2 \pi c / \omega$ inside the reststrahlen band.

To further investigate propagation properties of surface modes, the dispersion Eqs. (3) are numerically solved for four values of the vacuum gap over the operating range of the $\mathrm{CO}_{2}$ laser, and the obtained phase velocities $v_{\mathrm{ph}} \equiv c k_{0} / k^{\prime}$ and propagation lengths $L_{\text {prop }}=1 / 2 k^{\prime \prime}$ are plotted in Fig. 3 for L-modes (solid lines) and T-modes (dashed lines). The corresponding wavelengths $\lambda_{\text {lum }}$ and $\epsilon_{\text {lum }} \equiv \epsilon_{\mathrm{SiC}}^{\prime}\left(\lambda_{\text {lum }}\right)$ of the luminous L-modes for the three
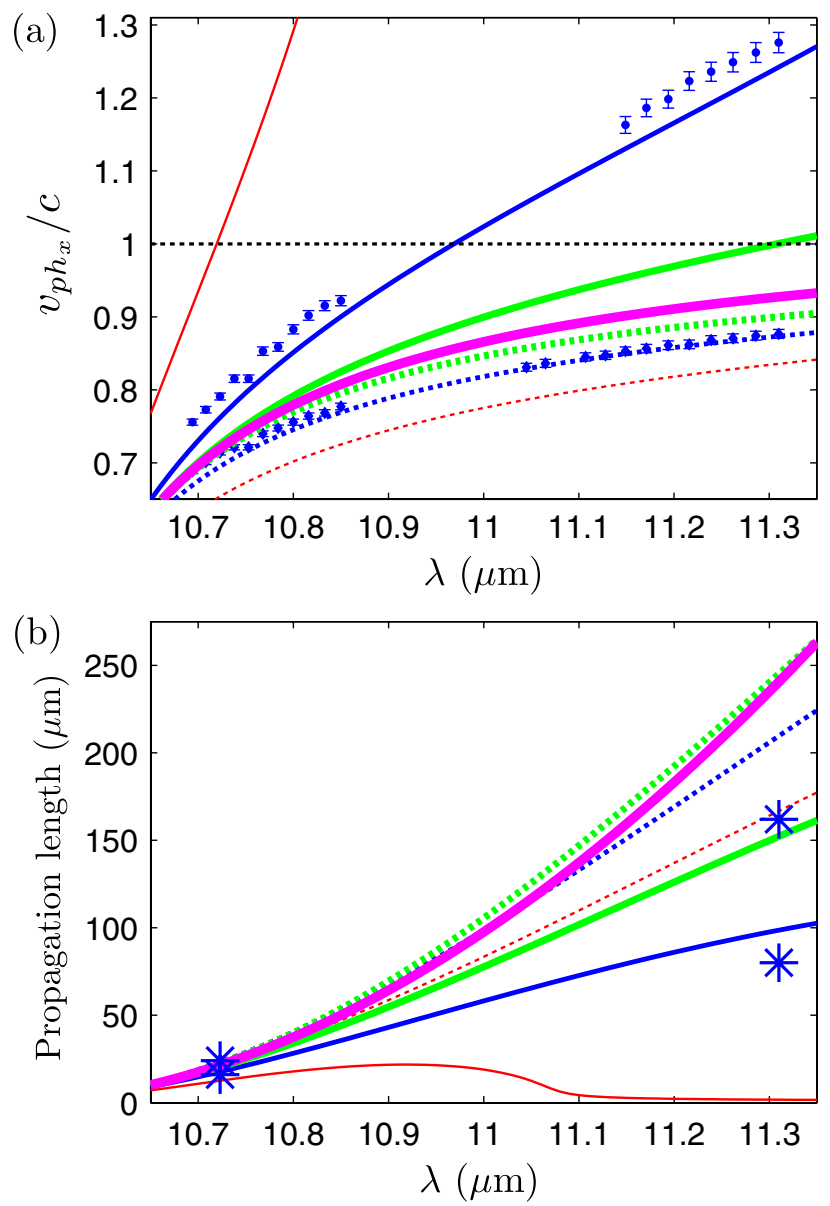

FIG. 3. (a) Theoretical (lines) and experimental (symbols) phase velocities plotted for different values of vacuum gap widths $2 a$. Experimental data were derived from $R\left(\theta_{i}\right)$ minima and correspond to $2 a=6 \mu \mathrm{m}$. (b) Theoretical plots of $L_{\text {prop }}=$ $1 / 2 k^{\prime \prime}$, with experimental estimates of propagation lengths for two frequencies, are included. Color scheme (and linewidths from thin to thick): $2 a=4 \mu \mathrm{m}$ (red), $6 \mu \mathrm{m}$ (blue), $9 \mu \mathrm{m}$ (green), and $\infty$ (single interface, magenta). Solid lines: accelerating L-modes, dashed lines: deflecting T-modes.

finite values of the gap $2 a$ presented in Fig. 3 are as follows: $\lambda_{\text {lum }}=10.72 \mu \mathrm{m}$ and $\epsilon_{\text {lum }}=-2.05$ for $2 a=4 \mu \mathrm{m}$; $\lambda_{\text {lum }}=10.97 \mu \mathrm{m}$ and $\epsilon_{\text {lum }}=-3.75$ for $2 a=6 \mu \mathrm{m}$; $\lambda_{\text {lum }}=11.31 \mu \mathrm{m}$ and $\epsilon_{\text {lum }}=-7.14$ for $2 a=9 \mu \mathrm{m}$. This trend confirms the previously stated general rule: as the gap is increased, a redshifting of the wavelength $\lambda_{\text {lum }}$ of the luminous L-mode toward $\lambda_{\mathrm{TO}}$ occurs. Naturally, the single-interface structure $(2 a=\infty)$ does not support any luminous modes.

Another important implication of the results plotted in Fig. 3(b) is that reducing the gap decreases the propagation length of the luminous L-mode from $L_{\text {prop }} \approx 150 \mu \mathrm{m}$ for $2 a=9 \mu \mathrm{m}$ to $L_{\text {prop }} \approx 15 \mu \mathrm{m}$ for $2 a=4 \mu \mathrm{m}$ because of the resistive losses in $\mathrm{SiC}$. The resistive quality factor of the mode can be evaluated from the propagation length 
according to $Q_{\text {res }} \approx\left(c / v_{g}\right) \times\left(k_{0} L_{\text {prop }}\right)$, where $v_{g}$ is the group velocity of the accelerating mode that can be calculated from the data presented in Fig. 3(a), according to $v_{g}=v_{\mathrm{ph}} /\left(1+\lambda / v_{\mathrm{ph}} \partial v_{\mathrm{ph}} / \partial \lambda\right)$. The quantity $Q_{\text {res }}$ determines the fill time of the structure according to $T_{\text {fill }} \approx$ $\left(Q_{\text {res }} / 2\right) \omega^{-1}$ if the following two simplifying assumptions are made: (i) beam-loading $Q_{\text {beam }}=Q_{\text {res }}$, and (ii) the structure is critically coupled [25]. Based on the properties of the luminous L-modes, only modest values of $Q_{\text {res }} \sim 500-1000$ are expected, which translates into $T_{\text {fill }} \sim 1.5 \mathrm{ps}-3.0 \mathrm{ps}$. Such pulse durations are presently achievable [3,4] with $\mathrm{CO}_{2}$ lasers.

An important implication of relatively high losses for accelerator applications is that high beam loading in a multibunch format [2], with the macrobunch duration roughly matched to $T_{\text {fill }}$, is required to maintain high overall efficiency of the SWABSiC. Another important implication of the highly restricted aperture of the SWABSiC is the importance of transverse wakes: a positive feedback loop resulting in a BBU instability develops when a vertically displaced microbunch excites a T-mode (transverse wake), which then further deflects the next (trailing) microbunch. Longitudinal wakes arising due to the excitation of L-modes contribute to energy spread within a macrobunch in the SWABSiC operated in the high beam-loading regime. Therefore, dispersion and loss characteristics of both $\mathrm{L}$ - and T-modes are crucial for determining the effects of the BBU instability and the beam-induced energy spread on a macrobunch. Accurate experimental analysis of these characteristics of L- and T-modes is necessary before implementing an accelerator. As demonstrated below, Land T-modes can be directly excited using a tunable laser as illustrated in Fig. 2. The modes' dispersion relations $\omega$ vs $k_{x}$ (or, alternatively, $v_{\text {ph }}$ vs $\lambda$ as shown in Fig. 3) can be mapped out by changing the incident angle $\theta_{i}$ of the laser beam.

\section{EXPERIMENTAL EXCITATION OF ACCELERATING AND DEFLECTING WAKES OF THE SWABSiC}

A schematic of the experimentally fabricated SWABSiC is shown in Fig. 2. Thin layers of $\operatorname{SiC}(1.7 \mu \mathrm{m})$ were epitaxially grown [21] on high-resistivity 5 -mm-thick $\mathrm{Si}$ slabs. Silicon is chosen for its compatibility with $\mathrm{SiC}$ growth and robustness to thermal and mechanical stress. One slab was fabricated by ISP Optics into a planar prism to couple mid-IR radiation into the accelerating structure. The high-index prism $\left(n_{\mathrm{Si}}=3.42\right)$ is required for the excitation of surface waves. The prism's dimensions indicated in Fig. 2 are as follows: the height is $1.78 \mathrm{~mm}$, and the angles of the in-coupling and out-coupling faces are $15^{\circ}$ and $28^{\circ}$, respectively. Using Snell's law, it is calculated that $\theta_{i} \equiv \theta_{0 i}=6.8^{\circ}$ (corresponding to total internal reflection at the $\mathrm{Si} /$ air interface) is required for exciting luminous wakes. The final SWABSiC structure with the
$2 a=6 \mu \mathrm{m}$ air gap is obtained by spin coating a polymer film of the same thickness onto the lower slab, followed by selective polymer removal and placing the two sides in contact as shown in Fig. 2.

The excitation and detection of the surface waves (both $\mathrm{L}$ - and T-modes) were carried out using a line-tunable ${ }^{13} \mathrm{CO}_{2}$ continuous wave laser (Access Laser Co.) by measuring the intensity of the reflected beam as a function of incident angle $\theta_{i}$ and wavelength $\lambda$. The typical incident power was $400 \mathrm{~mW}$. A 15 -cm-focal length ZnSe lens was used to focus the 4.5-mm-diameter IR beam. Measurements were carried out with $0.5^{\circ}$ angular step size for six selected wavelengths listed in Fig. 4. The reflected beam (R-beam) propagates along the following optical path: (i) transmission from air into the in-coupling face and through the $\mathrm{Si}$ prism, (ii) reflection from the $\mathrm{SiC}$ surface, and (iii) transmission through the prism into air via the out-coupling face. A minimum in the intensity of the R-beam [referred to as reflectivity $R\left(\theta_{i}, \lambda\right)$ in Fig. 4 and elsewhere] is used as the diagnostic of surface wave excitation. In the absence of $\mathrm{SiC}$, the reflectivity $R\left(\theta_{i}, \lambda\right)$ is expected to be essentially angle independent for $\theta_{i}>\theta_{0 i}$ (indicated by red line and stars in Fig. 4 for $\lambda=10.723 \mu \mathrm{m})$ because of total internal reflection (TIR) from the Si/air interface. The prism is designed to maintain an angular separation of at least $4^{\circ}$ between the R-beam and the specularly reflected beam (SR-beam)

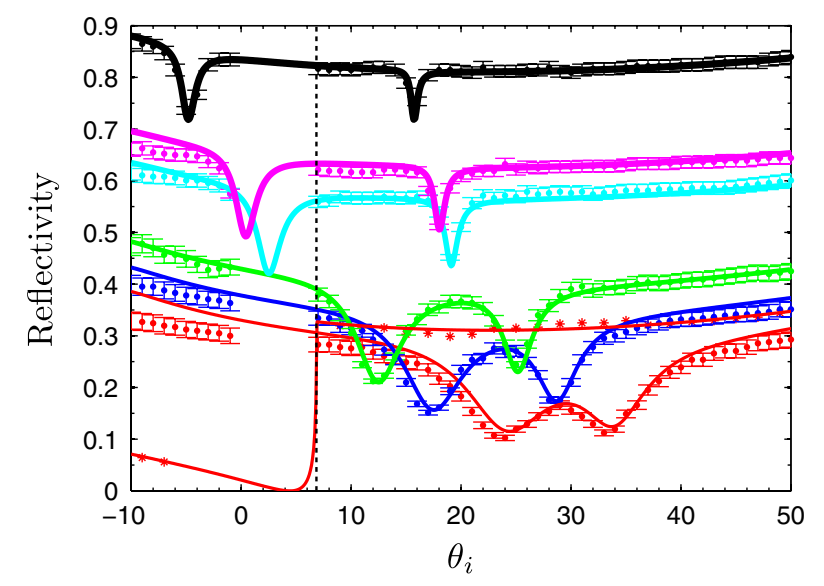

FIG. 4. Experimentally measured (dots) and theoretically calculated (lines) reflectivity spectra $R\left(\theta_{i}, \lambda_{k}\right)$ from the SWABSiC structure with the $2 a=6 \mu \mathrm{m}$ air gap. Colors (and linewidths from thin to thick) represent the wavelengths of the line-tunable ${ }^{13} \mathrm{CO}_{2}$ laser: $\lambda_{1}=10.723 \mu \mathrm{m}$ (red), $\lambda_{2}=10.784 \mu \mathrm{m}$ (blue), $\lambda_{3}=10.850 \mu \mathrm{m} \quad$ (green), $\quad \lambda_{4}=11.065 \mu \mathrm{m} \quad$ (cyan), $\quad \lambda_{5}=$ $11.128 \mu \mathrm{m}$ (magenta), and $\lambda_{6}=11.310 \mu \mathrm{m}$ (black). For clarity, the spectra are vertically offset by the value $\lambda_{k}(\mu \mathrm{m})-$ $\lambda_{1}(\mu \mathrm{m})$. A reflection spectrum from a bare prism (no $\mathrm{SiC}$ ) is also included for $\lambda_{1}$; theory (red solid line reaching zero) and experiment (red stars) are given. The dashed line at $\theta_{0 i}=6.8^{\circ}$ indicates TIR corresponding to the excitation of the luminous L-mode. 
produced at the in-coupling surface. This ensures that the SR-beam does not contribute to $R\left(\theta_{i}, \lambda\right)$ for any incident angle.

Experimentally measured reflectivity spectra $R\left(\theta_{i}, \lambda\right)$ are shown in Fig. 4 as dots, together with corresponding theoretical plots (solid lines) for six laser wavelengths (see caption for details). From Snell's law, larger $\theta_{i}$ correspond to larger values of $k_{x}^{\prime}$ and, therefore, smaller phase velocity. Laser coupling to surface waves is manifested as reflection minima. For every wavelength in the $10.723 \mu \mathrm{m}<\lambda<$ $11.310 \mu \mathrm{m}$ range, both L-modes (smaller $\theta_{i}$ ) and T-modes (larger $\theta_{i}$ ) are observed as distinct dips of $R\left(\theta_{i}, \lambda\right)$. Excellent agreement between theory and experiment is achieved by accounting for all relevant loss mechanisms applicable to the laser beam: surface wave excitation, reflections at the in-coupling and out-coupling faces of the prism, and two-photon absorption in Si. The experimental measurements indeed confirm that the T-modes are subluminous for all wavelengths whereas the L-mode can be luminous, subluminous, or superluminous, depending on the wavelength.

One drawback of the present prism design is that the luminous mode, which is excited at $\theta_{i}=\theta_{i 0}$, is not accessible because the direction of the R-beam is within a few degrees of the incident beam, causing feedback into the laser amplification chain. This results in the angular "data gap" in the $0<\theta_{i}<\theta_{0 i}$ angular range. Face angles of the prism can be redesigned to avoid this effect. Nevertheless, our experimental demonstration of subluminous and superluminous accelerating wakes clearly shows the possibility of designing resonant SWABSiC structures for accelerating relativistic electron beams.

Experimental data presented in Fig. 4 enables the extraction of both phase velocities (from the spectral positions of the reflectivity dips) and propagation lengths $L_{\text {prop }}$ (from the angular width of the dips) of the modes. The prism provides longitudinal wave numbers $k_{x}=$ $k_{0} n_{\mathrm{Si}} \sin \theta_{\mathrm{Si}}$, where $\theta_{\mathrm{Si}}$ is the incident angle within $\mathrm{Si}$ (and is related through Snell's law to the laser's incidence angle $\theta_{i}$ from the prism normal, as indicated in Fig. 2). Recalling that $v_{\mathrm{ph}}=\omega / k_{x}$ and $k_{0}=\omega / c$, rearranging the equation for $k_{x}$ yields $v_{\mathrm{ph}} / c=1 /\left[n_{\mathrm{Si}} \sin \left(\theta_{\mathrm{Si}}\right)\right]$. Using Fig. 4 results and additional data, normalized phase velocities [Fig. 3(a)] are derived from reflectivity minima originating from surface waves.

The experimental values of $L_{\text {prop }}^{\text {exp }}$ can be estimated as $L_{\text {prop }}^{\exp } \approx 1 / \Delta k_{x}$, where $\Delta k_{x}=2 \pi n_{\mathrm{Si}} / \lambda \Delta \sin \theta$ is determined by the FWHM angular width of the reflectivity curve, and $\theta$ is the incidence angle onto the $\mathrm{Si} / \mathrm{SiC}$ interface. It is found that the measured $L_{\text {prop }}^{\exp }$ for $2 a=6 \mu \mathrm{m}$ is in general agreement with theoretically predicted $L_{\text {prop }}$ plotted with blue lines in Fig. 3(b). For example, $L_{\text {prop }}^{\exp }$ is indeed found to vary considerably depending on the wavelength, from $L_{\text {prop }}^{\exp }\left(\lambda_{1}=10.723 \mu \mathrm{m}\right)=16 \mu \mathrm{m}$ to $L_{\text {prop }}^{\exp }\left(\lambda_{6}=11.310 \mu \mathrm{m}\right)=80 \mu \mathrm{m}$ for L-modes, and from $L_{\text {prop }}^{\exp }(10.723 \mu \mathrm{m})=24 \mu \mathrm{m}$ to $L_{\text {prop }}^{\exp }(11.310 \mu \mathrm{m})=$ $162 \mu \mathrm{m}$ for T-modes. Propagation lengths can be further used for calculating the quality factors of the modes. To our knowledge, these measurements represent the first experimental characterization of transverse and longitudinal modes of a laser-driven accelerating structure.

\section{ACCELERATOR AND BEAM DIAGNOSTICS APPLICATIONS OF THE SWABSiC}

In this section we consider the implications of the electromagnetic properties of SWABSiC for designing laserdriven accelerators and infrared radiation sources. For accelerator applications, optical properties of SWABSiC determine the appropriate formats of the accelerated microbunches, microbunch trains, and laser pulses delivering energy to the structure. For example, the fill time $T_{\text {fill }}=$ $Q \omega^{-1}$ of the accelerating structure with the quality factor $Q$ determines the optimal duration of the laser pulse powering the accelerator. Another important accelerator parameter is the loss factor of the acceleration mode per unit of acceleration length $\kappa$, which is defined as $\kappa_{\|}=$ $E_{x}^{2} / 4 U$, where $E_{x}$ is the peak on-axis acceleration field and $U$ is the stored energy of the mode per unit of acceleration length. The loss parameter enables estimating the required energy storage per unit length $U$ that is needed to maintain a given acceleration gradient. Given the fill time of the structure, it also enables the calculation of the laser power $P$ required to maintain such acceleration gradient. With an acceleration structure length $L_{x}$ and width $W_{z}$, we estimate that $P \sim E_{x}^{2} L_{x} /\left(4 \kappa T_{\text {fill }}\right)$.

Under a set of simplifying assumptions, the order of magnitude for $\kappa$ can be evaluated. Assuming that most of the stored energy is contained inside the $\mathrm{SiC}$ slabs, and that the penetration depth into $\mathrm{SiC}$ slabs is $l_{\text {skin }} \sim \lambda / 8 \pi$, the stored energy per unit length can be estimated as

$$
U \approx \frac{E_{x}^{2} W_{z}}{32 \pi^{2}} \frac{\partial\left(\omega \epsilon_{\mathrm{SiC}}\right)}{\partial \omega} .
$$

A rough estimate of $\partial\left(\omega \epsilon_{\mathrm{SiC}}\right) / \partial \omega \approx 100$ enables the following order-of-magnitude estimate of $\kappa \sim\left(W_{z} \lambda\right)^{-1}$, which can be recast in the following familiar [19] form: $\kappa \sim\left(100 \lambda / W_{z}\right) \times \lambda^{-2}[\mathrm{~cm}] \mathrm{V} /(\mathrm{pC} \mathrm{m})$. For example, assuming $W_{z}=1 \mathrm{~mm}$ and $\lambda=11 \mu \mathrm{m}$, we obtain $\kappa \sim$ $1 \mathrm{MV} /(\mathrm{pCm})$, which translates into $U=250 \mathrm{~mJ} / \mathrm{m}$ for an $E_{z}=1 \mathrm{GeV} / \mathrm{m}$ accelerating gradient. For a single structure of length $L=1 \mathrm{~cm}$ with a fill time of $T_{\text {fill }}=$ $3 \mathrm{ps}$, the total stored energy of $2.5 \mathrm{~mJ}$ requires a laser power of order $P_{1}=1 \mathrm{GW}$. If such a structure is utilized as a single stage of a larger laser-driven accelerator, then the single-stage gain is of order $10 \mathrm{MeV}$. Assuming the efficiency of energy recovery by the accelerated beam to be $\eta=40 \%$, the total charge of a macrobunch can be estimated to be of order $Q=10 \mathrm{pC}$. Note that $N=100$ stages would impart a $1 \mathrm{GeV}$ energy gain to the accelerated bunch 
while requiring the total laser power of order $P_{\text {tot }}=$ $0.1 \mathrm{TW}$, which is entirely within reach of the existing short-pulse $\mathrm{CO}_{2}$ laser systems.

Finally, we discuss the possibility of using the SWABSiC structure as a source of coherent infrared radiation that can be used for characterization of the electron bunch's length and transverse size. Such diagnostics are particularly desirable for ultrashort low-emittance bunches produced by laser-plasma accelerators. It is well established $[17,26,27]$ that, regardless of the specific mechanism of radiation generation (e.g., synchrotron, transition, or undulator), the spectral intensity of far-field radiation emitted by a bunch of $N_{e}$ can be expressed as the sum of coherent and incoherent components:

$$
I_{N_{e}}(\omega)=I_{1}(\omega)\left[1+\left(N_{e}-1\right) f(\omega)\right],
$$

where $I_{1}(\omega)$ is the spectral intensity of radiation emitted by a single electron, and $f(\omega)$ is the form factor determined by the dimensions of the bunch. For a Gaussian bunch with rms dimensions $\sigma_{x, y, z}$ whose centroid is located in the midplane of the SWABSiC structure, it can be demonstrated that the form factor of the coherent radiation that results from the excitation of the $\mathrm{L}$-mode is proportional to

$$
f(\omega) \propto e^{-\left(k \sigma_{z} \cos \theta_{b}\right)^{2}} e^{-\left(k \sigma_{y} \sin \theta_{b}\right)^{2}},
$$

where $\theta_{b}(\omega)$ is the frequency-dependent Cherenkov angle of the radiation coherently emitted in the $x-z$ plane by the bunched beam. No coherent T-mode is generated by such a midplane centered bunch. Quantitative information about the size of the bunch can be obtained from the angular dependence of coherent radiation using Eq. (6). Because $k$ is of order $1 \mu \mathrm{m}$, such diagnostics are particularly suitable for bunches with micron-scale dimensions. Moreover, micron-scale displacements of the beam's centroid from the midplane can be detected by measuring far-field coherent radiation due to the excitation of the T-modes. Such radiation arises only for finite centroid displacements in the $y$ direction, and it is spectrally distinguishable from radiation due to the L-modes.

\section{CONCLUSIONS}

In conclusion, we have theoretically and experimentally investigated optical properties of the surface wave accelerator based on silicon carbide (SWABSiC). The SWABSiC structure consists of two layers of the negative permittivity material $\mathrm{SiC}$, separated by a vacuum gap. The structure can be used either as a laser-driven accelerator or as a source of coherent infrared radiation. In the latter case, the coherent radiation produced by an ultrashort relativistic bunch can be used for characterizing the transverse and longitudinal dimensions of the bunch, as well as the centroid's position with respect to the midplane in the vacuum gap. By coupling infrared light from a carbon dioxide laser through the silicon prism into the SWABSiC, we excited two types of surface modes of the structure-the longitudinal (accelerating) mode and transverse (deflecting) mode. Their phase velocities and quality factors were experimentally measured and compared with theoretical calculations. An example of a SWABSiC driven by a highpower short-pulse $\mathrm{CO}_{2}$ laser was presented. The promise of the structure for beam diagnostics was also discussed.

\section{ACKNOWLEDGMENTS}

The authors thank Chris Fietz for useful discussions and simulation assistance. This work was supported by U.S. DOE Grant No. DE-FG02-04ER41321.

[1] D. H. Whittum, AIP Conf. Proc. 472, 72 (1999).

[2] R. B. Palmer et al., AIP Conf. Proc. 130, 234 (1985).

[3] D. Haberberger, S. Tochitsky, and C. Joshi, Opt. Express 18, 17865 (2010).

[4] M. N. Polyanskiy, I. V. Pogorelsky, and V. Yakimenko, Opt. Express 19, 7717 (2011).

[5] W. Gai, P. Schoessow, B. Cole, R. Konecny, J. Norem, J. Rosenzweig, and J. Simpson, Phys. Rev. Lett. 61, 2756 (1988).

[6] J. Rosenzweig, A. Murokh, and C. Pellegrini, Phys. Rev. Lett. 74, 2467 (1995).

[7] L. C. Steinhauer and W. D. Kimura, Phys. Rev. ST Accel. Beams 6, 061302 (2003).

[8] X. E. Lin, Phys. Rev. ST Accel. Beams 4, 051301 (2001).

[9] C.-K. Ng, R. J. England, L.-Q. Lee, R. Noble, V. Rawat, and J. Spencer, Phys. Rev. ST Accel. Beams 13, 121301 (2010).

[10] A. Mizrahi and L. Schachter, Phys. Rev. E 70, 016505 (2004).

[11] B. M. Cowan, Phys. Rev. ST Accel. Beams 6, 101301 (2003).

[12] B. M. Cowan, Phys. Rev. ST Accel. Beams 11, 011301 (2008).

[13] R. B. Yoder and J. B. Rosenzweig, Phys. Rev. ST Accel. Beams 8, 111301 (2005).

[14] M. A. Shapiro, W. J. Brown, I. Mastovsky, J. R. Sirigiri, and R. J. Temkin, Phys. Rev. ST Accel. Beams 4, 042001 (2001).

[15] G. Shvets and S. Kalmykov, AIP Conf. Proc. 737, 983 (2004).

[16] S. Kalmykov, O. Polomarov, D. Korobkin, J. Otwinowski, J. Power, and G. Shvets, Phil. Trans. R. Soc. A 364, 725 (2006).

[17] C. P. Neuman, W. S. Graves, and P. G. O'Shea, Phys. Rev. ST Accel. Beams 3, 030701 (2000).

[18] W. G. Spitzer, D. Kleinman, and D. Walsh, Phys. Rev. 113, 127 (1959).

[19] C. B. Schroeder, D. H. Whittum, and J. S. Wurtele, Phys. Rev. Lett. 82, 1177 (1999).

[20] C. E. Weitzel, J. W. Palmour, C. H. Carter Jr., K. Moore, K. K. Nordquist, S. Allen, C. Thero, and M. Bhatnagar, IEEE Trans. Electron Devices 43, 1732 (1996).

[21] M. J. Hernandez, G. Ferro, T. Chassagne, J. Dazord, and Y. Monteil, J. Cryst. Growth 253, 95 (2003). 
[22] M. B. J. Wijesundara, G. Valente, W. R. Ashurst, R. T. Howe, A.P. Pisano, C. Carraro, and R. Maboudian, J. Electrochem. Soc. 151, C210 (2004).

[23] S. Rajasekhara, B.H. Neuner III, C. A. Zorman, N. Jegenyes, G. Ferro, G. Shvets, P. J. Ferreira, and D. Kovar, Appl. Phys. Lett. 98, 191904 (2011).

[24] Cubic Silicon Carbide $(\beta-S i C)$, Handbook of Optical Constants of Solids, edited by S. A. Alterovitz, J. A.
Woollam, and E.D. Palik (Academic, New York, 1991).

[25] B. Neuner III, D. Korobkin, C. Fietz, D. Carole, G. Ferro, and G. Shvets, Opt. Lett. 34, 2667 (2009).

[26] H. Motz, J. Appl. Phys. 22, 527 (1951).

[27] T. Watanabe et al., Nucl. Instrum. Methods Phys. Res., Sect. A 437, 1 (1999). 